Decision Making in Manufacturing and Services

VOL. $1 \bullet 2007 \bullet$ No. 1-2 • PP. 49-57

\title{
The Errors-in-Variable Model in the Optimal Portfolio Construction
}

\author{
Anna Czapkiewicz*, Małgorzata Machowska*
}

\begin{abstract}
In the paper we consider a modification of Sharpe's method used in classical portfolio analysis for optimal portfolio building. The conventional theory assumes there is a linear relationship between asset's return and market portfolio return, while the influence of all the other factors is not included. We propose not to neglect them any more, but include them into a model. Since the factors in question are often hard to measure or even characterize, we treat them as a disturbances on random variables used by classical Sharpe's method.

The key idea of the paper is the modification of the classical approach by application of the errors-in-variable model. We assume that both independent (market portfolio return) as well as dependent (given asset's return) variables are randomly distributed values related with each other by linear relationship and we build the model used for parameters' estimation.

To verify the model, we performed an analysis based on archival data from Warsaw Stock Exchange. The results are also included.
\end{abstract}

Keywords: maximum likelihood method,errors-in-variables model, portfolio, simulation study Journal of Economics Literature Classification: G11

Received/Revised: 15 January 2007/05 May 2007

\section{CLASSIC SHARPE'S MODEL}

The ratio of return we determine as relative increase of asset's price in time i.e. $R_{t}^{k}=\frac{S_{t}^{k}-S_{t-1}^{k}}{S_{t-1}^{k}}$, with $S_{t}^{k}$ being the price of $k$-th asset at time $t$.

In classic Sharpe's model return of $k$-th $R^{k}$ is explained by market portfolio return $R^{m}$ trough characteristic line equation according to the relationship: $R^{k}=\beta_{k}\left(R^{m}-r_{f}\right)+r_{f}+\varepsilon_{k}$, where $r_{f}$ is riskless rate of interest.

\footnotetext{
* AGH University of Science and Technology, Faculty of Management, Cracow. E-mail: gzrembie@cyf-kr.edu.pl, mmachow@agh.edu.pl.
} 
Knowing the market sensitivity $\beta_{k}$ of $k$-th asset we receive the formula for the expected value and the variance of portfolio return ratio:

$$
\begin{aligned}
E\left(R_{p}\right) & =\beta\left(E\left(R^{m}\right)-r_{f}\right)+r_{f}, \\
D^{2}\left(R_{p}\right) & =\beta^{2} D^{2}\left(R^{m}\right)+\sum_{k=1}^{k=K} x_{k}^{2} D^{2}\left(\varepsilon_{k}\right)
\end{aligned}
$$

with $\beta=\sum_{k=1}^{k=K} x_{k} \beta_{k}$; where $x_{k}-k$-th stock's share in investment portfolio. In this model the $\beta_{k}$ coefficient is estimated by least square method.

\section{MODIFIED SHARPE'S MODEL}

\subsection{MODEL WITH REPLICATIONS}

Let us consider the situation, in which both: dependent variable, being the certain asset's surplus return ratio and denoted $R^{k}$ as well as independent variable - the market portfolio surplus return ratio $R^{m}$ are disturbed observations related with each other by linear equation, i.e.:

$$
R^{m}-r_{f}=\xi+\varepsilon ; \quad R^{k}-r_{f}=\beta_{k} \xi+\alpha_{k}+\delta_{k}
$$

If we assume that random variables $\varepsilon, \delta_{k}$ have normal distribution with unknown parameters then model (3) is unidentifable, what means that there are not estimation methods allowing to determine consistent estimators of the $\beta_{k}$ parameters (Reirsol, 1950). The parameter $\beta_{k}$ has been shown to be identifiable only under various sets of assumptions, for example: the variance of one disturbance is known or, more commonly, the variance ratio is known. There are several methods of estimating $\beta_{k}$ and $\alpha_{k}$ in such cases (Markowitz, 1952).

From the practical point of view, we are most interested in an approach which leads to estimating the parameters in the case when all parameters have to be considered as unknown. In that case, replication of measurement of each pair of observation: dependent and independent; $m$ times overcomes the nonidentifiability (Bunke, Bunke, 1989).

The following approach was therefore proposed: returns of given asset and market portfolio are analysed within periods of $t$. To be more specific the period of one month has been assumed as $t$.

Let $n$ denotes number of historical months, $m$ number of monthly returns during given month and $K$ number of assets in portfolio. Let $R_{i j}^{m}$ and $R_{i j}^{k}$ are $j$-th monthly return ratios in the $i$-th month of portfolio market and $k$-th asset, respectively.

Let $X_{i j}, Y_{i j}^{k}$ denote: $X_{i j}=R_{i j}^{m}-r_{f}$ and $Y_{i j}^{k}=R_{i j}^{k}-r_{f}$.

The following relationships for surplus return ratios were considered:

$$
X_{i j}=\xi_{i j}+\varepsilon_{i j}, \quad Y_{i j}^{k}=\beta_{k} \xi_{i j}+\alpha_{k}+\delta_{i j}^{k}, \quad i=1, \ldots, n, \quad j=1, \ldots, m, \quad k=1, \ldots, K
$$


It has been assumed that $\delta_{i j} \sim N\left(0, \sigma_{\delta}^{2 k}\right), \xi_{i j}^{k} \sim N\left(s_{i}, \sigma_{s}^{2}\right), \varepsilon_{i j} \sim N\left(0, \sigma_{\varepsilon}^{2}\right)$. All the parameters of aforementioned distributions are unknown.

Now the issue of optimal portfolio choice is finding: $\max \left[E\left(R_{p}\right)-\lambda D^{2}\left(R_{p}\right)\right]$ under conditions: $x_{k} \geq 0, \quad \sum_{k=1}^{k=K} x_{k}=1$ with:

$$
E\left(R_{p}\right)=\sum_{k=1}^{k=K} x_{k}\left(\beta_{k} s+r_{f}\right), \quad D^{2}\left(R_{p}\right)=\left(\sum_{k=1}^{k=K} x_{k} \beta_{k}\right)^{2} \sigma_{s}^{2}+\sum_{k=1}^{k=K} x_{k}^{2} \sigma_{\delta_{k}}^{2}
$$

The unknown parameters sis the expected value for distribution of monthly returns of market portfolio during the period of the prognosis.

\section{ESTIMATION OF UNKNOWN PARAMETERS}

\subsection{FIRST STEP - ESTIMATION OF PARAMETERS FOR GIVEN ASSET}

For given $k$ in model (4) the number of unknown parameters to be estimated increases with $\mathrm{n}$. Unknown parameters are: $\beta_{k}, \alpha_{k}, \sigma_{\delta_{k}}^{2}, \sigma_{s}^{2}, \sigma_{\varepsilon}^{2}, s_{1}, \ldots, s_{n}$. It can be shown, that in this model the maximum likelihood estimators have normal distribution asymptotically with respect to $m$ and $n$ (Dolby, 1976).

Let: $X_{i .}=\frac{1}{m} \sum_{j=1}^{m} X_{i j}, Y_{i .}^{k}=\frac{1}{m} \sum_{j=1}^{m} Y_{i j}^{k}, X_{. .}=\frac{1}{n} \sum_{j=1}^{m} X_{i .}, Y_{. .}^{k}=\frac{1}{n} \sum_{j=1}^{n} Y_{i .}^{k}$ and subsequently:

$$
\begin{aligned}
w_{x x} & =\frac{1}{m n} \sum_{i=1}^{n} \sum_{j=1}^{m}\left(X_{i j}-X_{i .}\right)^{2} & s_{x x} & =\frac{1}{n} \sum_{i=1}^{n} \sum_{j=1}^{m}\left(X_{i .}-X_{. .}\right)^{2} \\
w_{y y}^{k} & =\frac{1}{m n} \sum_{i=1}^{n} \sum_{j=1}^{m}\left(Y_{i j}^{k}-Y_{i .}^{k}\right)^{2} & s_{y y}^{k} & =\frac{1}{n} \sum_{i=1}^{n} \sum_{j=1}^{m}\left(Y_{i .}^{k}-Y_{. .}\right)^{2} \\
w_{x y}^{k} & =\frac{1}{m n} \sum_{i=1}^{n} \sum_{j=1}^{m}\left(X_{i j}-X_{i .}\right)\left(Y_{i j}^{k}-Y_{i .}^{k}\right) & s_{x y}^{k} & =\frac{1}{n} \sum_{i=1}^{n} \sum_{j=1}^{m}\left(X_{i .}-X_{. .}\right)\left(Y_{i .}^{k}-Y_{. .}\right)
\end{aligned}
$$

the maximum likelihood estimator of $\beta_{k}$ and $\alpha_{k}$ coefficients have forms:

$$
\hat{\beta}_{k}=\frac{w_{y y}^{k} s_{x x}-w_{x x} s_{y y}^{k}-\sqrt{\Delta}}{2\left(w_{x y}^{k} s_{x x}-w_{x x} s_{x y}^{k}\right)} \quad \hat{\alpha}_{k}=Y_{i j}^{k}-\hat{\beta}_{k} X_{i j}
$$

where

$$
\Delta=\left(w_{x x} s_{y y}^{k}-s_{x x} w_{y y}^{k}\right)^{2}-4\left(s_{x y}^{k} w_{y y}^{k}-s_{y y}^{k} w_{x y}^{k}\right)\left(s_{x x} w_{x y}^{k}-s_{x y}^{k} w_{x x}\right) .
$$

The estimators of all the other values are expressed in terms of $\hat{\beta}_{k}$ (Cox, 1976). These estimators have forms of: 


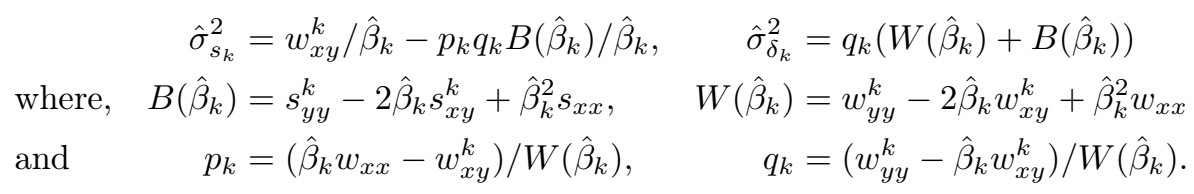

\subsection{SECOND STEP - ESTIMATION OF UNKNOWN PARAMETERS FOR THE WHOLE MODEL}

For given $j$ we construct vectors: $x_{j}^{T}, y_{j}^{k T}$ as:

$$
x_{j}^{T}=\left(X_{1 j}, \ldots, X_{n j}\right), \quad y_{j}^{k T}=\left(Y_{1 j}^{k}, \ldots, Y_{n j}^{k}\right), \quad k=1, \ldots, K
$$

The random variables $\mu_{j}=\left(x_{j}^{T}, y_{j}^{1 T}, y_{j}^{2 T}, \ldots, y_{j}^{K T}\right)$ are independent with the same normal distribution and the vector of expected values $\mu=\left(s^{T},\left(\beta_{1} s+\alpha_{k}\right)^{T}, \ldots,\left(\beta_{K} s+\right.\right.$ $\left.\alpha_{k}\right)^{T}$ ) where: $s^{T}=\left(s_{1}, \cdots, s_{n}\right)$ and with the covariance matrix $\mathbf{V}$, where

$$
V=\left[\begin{array}{llll}
\sigma_{s}^{2}+\sigma_{\varepsilon}^{2} & \beta_{1} \sigma_{s}^{2} & \ldots & \beta_{K} \sigma_{s}^{2} \\
\beta_{1} \sigma_{s}^{2} & \beta_{1}^{2} \sigma_{s}^{2}+\sigma_{\delta_{1}}^{2} & \ldots & \beta_{1} \beta_{K} \sigma_{\varepsilon}^{2} \\
\vdots & \vdots & \ddots & \vdots \\
\beta_{K} \sigma_{s}^{2} & \beta_{1} \beta_{K} \sigma_{\varepsilon}^{2} & \ldots & \beta_{K}^{2} \sigma_{s}^{2}+\sigma_{\delta_{K}}^{2}
\end{array}\right] \otimes I_{n}=\sum \otimes I_{n}
$$

The logarithm of likelihood function for variable $\mu_{j}$ with unknown parameters of: $\beta_{1}, \ldots, \beta_{k}, \alpha_{1}, \ldots \alpha_{k}, \sigma_{s}^{2}, \sigma_{\varepsilon}^{2}, \sigma_{\delta_{1}}^{2}, \ldots, \sigma_{\delta_{K}}^{2}, s_{1}, \ldots s_{n}$, has a form of:

$$
\log L\left(\mu_{j}, \Psi\right)=C-\frac{m}{2} \log (\operatorname{det}(V))-\frac{1}{2} \sum_{j=1}^{m} d_{j} V^{-1} d_{j}
$$

where $d_{j}=\left(\mu_{j}-\mu\right)$ and $\Psi$ is the set of unknown parameters.

Let $V_{\psi}$ denote a matrix composed of matrix $\boldsymbol{V}$ elements after derivation with respect to $\psi$ parameters ( $\psi$ being the arbitrary element of $\Psi$ set). The $\mathbf{V}$ matrix is symmetric thus:

$$
\frac{\partial}{\partial \psi} \log L\left(\mu_{j}, \Psi\right)=m\left\{\frac{1}{2} \operatorname{tr}\left(P V_{\psi}\right)-d_{\psi}^{T} V^{-1} d\right\}
$$

with

$$
P=V^{-1}(D-V) V^{-1} \quad \text { and } \quad D=\frac{1}{m} \sum_{j=1}^{m} d_{j} d_{j}^{T} .
$$

It comes that:

$$
\begin{gathered}
d_{\alpha_{k}}^{T} V^{-1} d=0, \quad \frac{1}{2} \operatorname{Tr}\left(P V_{\beta_{k}}\right)-d_{\beta_{k}}^{T} V^{-1} d=0, \quad d_{s_{i}}^{T} V^{-1} d=0, \\
\operatorname{Tr}\left(P V_{\sigma_{\varepsilon}^{2}}\right)=0, \quad \operatorname{Tr}\left(P V_{\sigma_{\delta_{k}}^{2}}\right)=0, \quad \operatorname{Tr}\left(P V_{\sigma_{s}^{2}}\right)=0 .
\end{gathered}
$$

From equations $d_{\alpha_{k}}^{T} V^{-1} d=0$ and $d_{s_{i}}^{T} V^{-1} d=0$ we received relationships: 


$$
\alpha_{k}=Y_{. .}^{k}-\beta_{k} X_{. .}
$$

and

$$
s_{i}=\frac{X_{i .}+A_{1}\left(Y_{.}^{1}-Y_{. .}^{1}+\beta_{1} X_{. .}\right)+\ldots+A_{K}\left(Y_{.}^{K}-Y_{. .}^{K}+\beta_{K} X_{. .}\right)}{1+A_{1} \beta_{1}+\ldots+A_{K} \beta_{K}}
$$

where $A_{k}=\beta_{k} \sigma_{\varepsilon}^{2} / \sigma_{\delta_{k}}^{2}$.

From: $\operatorname{Tr}\left(P V_{\sigma_{\varepsilon}^{2}}\right)=0, \operatorname{Tr}\left(P V_{\sigma_{\delta_{k}}^{2}}\right)=0$, exploiting certain features of matrix algebra we obtain:

$$
\sigma_{\varepsilon}^{2}+\sigma_{s}^{2}=\sum_{i=1}^{n} \sum_{j=1}^{m} \frac{\left(X_{i j}-s_{i}\right)^{2}}{m n} \quad \sigma_{\delta_{k}}^{2}+\beta_{k}^{2} \sigma_{s}^{2}=\sum_{i=1}^{n} \sum_{j=1}^{m} \frac{\left(Y_{i j}-\beta_{k} s_{i}-\alpha_{k}\right)^{2}}{m n}
$$

From equations (7), (8) and relationship $\operatorname{Tr}\left(P V_{\sigma_{s}^{2}}\right)=0$ we calculated $\sigma_{\varepsilon}^{2}$ as:

$$
\sigma_{\varepsilon}^{2}=\sum_{k=1}^{K}\left(1-\frac{T^{k}\left(\beta_{k}\right)}{\sigma_{\delta_{k}}^{2}}\right) /\left(\frac{1}{2} \sum_{i=1}^{K} \sum_{k=1}^{K} \frac{T^{k l}\left(\beta_{k}, \beta_{l}\right)}{\sigma_{\delta_{k}}^{2} \sigma_{\delta_{l}}^{2}}-K \sum_{k=1}^{K} \frac{\beta_{k}^{2}}{\sigma_{\delta_{k}}^{2}}\right):
$$

with:

$$
\begin{gathered}
T^{k}\left(\beta_{k}\right)=\left(s_{y y}^{k}+w_{y y}^{k}\right)-2 \beta_{k}\left(s_{x y}^{k}+w_{x y}^{k}\right)+\beta_{k}^{2}\left(s_{x x}+w_{x x}\right) \\
T^{k l}\left(\beta_{k}, \beta_{l}\right)=\beta_{k}^{2}\left(s_{y y}^{l}+w_{y y}^{l}\right)-2 \beta_{k} \beta_{l}\left(s_{y y}^{k l}+w_{y y}^{k l}\right)+\beta_{l}^{2}\left(s_{y y}^{k}+w_{y y}^{k}\right) . \\
w_{y y}^{k l}=\frac{1}{n m} \sum_{i=1}^{n} \sum_{j=1}^{m}\left(Y_{i j}^{k}-Y_{i .}^{k}\right)\left(Y_{i j}^{l}-Y_{i .}^{l}\right) \quad s_{y y}^{k l}=\frac{1}{n} \sum_{i=1}^{n}\left(Y_{i .}^{k}-Y_{. .}^{k}\right)\left(Y_{i .}^{l}-Y_{. .}^{l}\right) .
\end{gathered}
$$

From relationships (8) eliminating $\sigma_{s}^{2}$ parameters, we received $k$ of square equations with $\sigma_{\varepsilon}^{2}$ as a variable. Applying the Viete's formulas for each of them we received the $\sigma_{\delta_{k}}^{2}$ in terms of $\sigma_{\delta_{1}}^{2}$

$$
\sigma_{\delta_{k}}^{2}=\frac{\beta_{k}^{2}}{\beta_{1}^{2}}\left(\sigma_{\delta_{1}}^{2}-T^{1}\left(\beta_{1}\right)-2 \beta_{1} w_{x y}^{1}\right)+T^{k}\left(\beta_{k}\right)+2 \beta_{k} w_{x y}^{k} .
$$

for $k=2, \ldots, K$.

Substituting $\sigma_{\delta_{k}}^{2}$ calculated according to (10) into equation (9), we received relationship between $\sigma_{\varepsilon}^{2}$ and $\sigma_{\delta_{1}}^{2}$. The parameter $\sigma_{\varepsilon}^{2}$ obtained in such a way we substitute into one of $k$ square equations, which gives a basis for calculating $\sigma_{\delta_{1}}^{2}$.

Ultimately the maximum of likelihood function depends only on unknown $\beta_{1}, \ldots, \beta_{K}$.

The last may be determined numerically starting from initial values equal to their estimators calculated in the first step. 


\section{FEATURES OF PROPOSED MODEL ANALYSED ON THE BASIS OF WARSAW STOCK EXCHANGE'S DATA}

For all the assets listed on Warsaw Stock Exchange from 2002 until 2006 we calculated $\beta$. It came out that using least squares method and maximum likelihood method (6) we receive different results: $\hat{\beta}$ and $\tilde{\beta}$, respectively. Moreover for majority of assets we observed strong time-variance of $\tilde{\beta}$ resulting from "hoops" of monthly returns. The aforementioned "hoops" are smoothed by $\hat{\beta}$. The data analysis was therefore performed in three phases.

\section{First phase}

All the assets were sorted out by their $\hat{\beta}$ in the decreasing order and divided into monthly portfolios containing subsequent nine stocks. As the market portfolio the portfolio being the base of WIG index was assumed. For each of them optimal assets' shares were determined using classical and modified method. Next, both real monthly profits for the month being the subject of prognosis were compared. Analysis was performed building portfolios for subsequent months of 2006 starting from January till November. For analysis, $\tilde{\beta}$ estimators calculated in the first step were taken. Slight differences in $\sigma_{s}^{2}$ estimated for various assets have been corrected by averaging. For estimation of unknown value of $s, \sum_{i=1}^{n} s_{i}$ was assumed. It can be shown that: $\sum_{i=1}^{n} s_{i}=x_{. .}$. The $\lambda$ was assumed at the level where there is not further diversification of the portfolio.

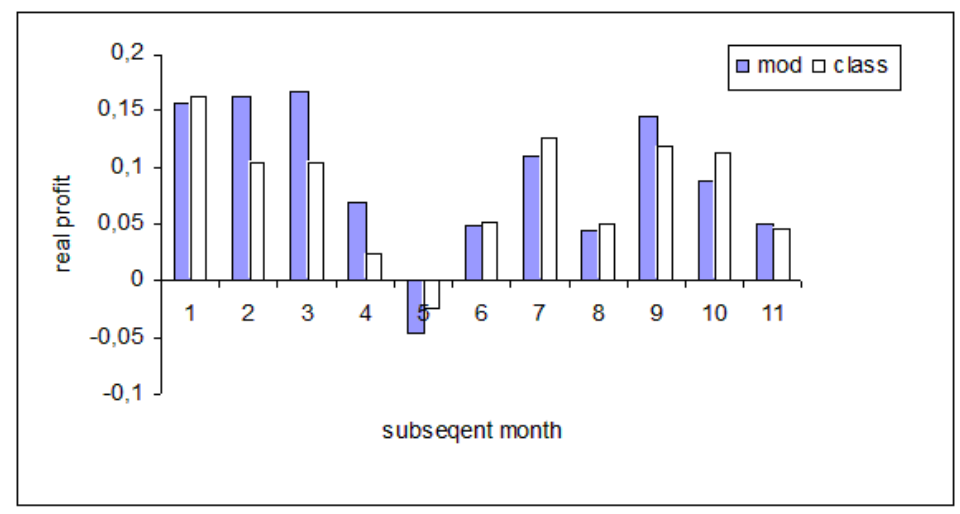

Fig. 1. Average monthly profits for portfolio built with classical and modified method

In Figure 1 we present average (of analysed 15 portfolios) real monthly profits for subsequent months for portfolios constructed using classical method (white bars) and modified one (grey bars).Using the classical portfolio construction strategy, the yearly return ratio of $119 \%$ was obtained while the modified one $-135 \%$. 


\section{Second phase}

A similar analysis was made using ordering assets listed on WSE with respect to $\beta$ calculated using modified method. Since such calculated $\tilde{\beta}$ for some companies exhibit strong time-variance the set of analysed stocks was reduced to that of the most "robust" $\tilde{\beta} s$. In such a way two portfolios containing nine assets were chosen. In Figures 2 and 3 we present the same data as in Figure 1, but for obvious reason there are raw data for both portfolios not their aggregates.

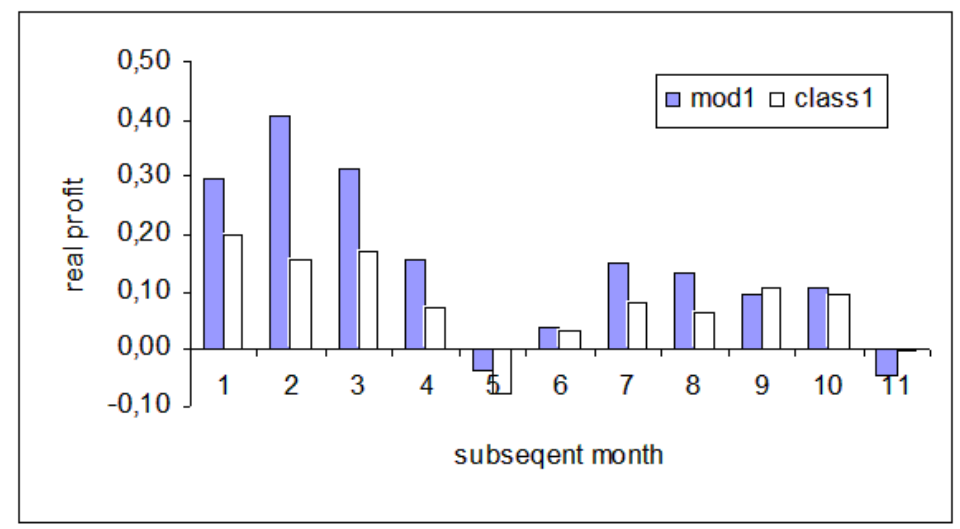

Fig. 2. Monthly profits for portfolio \#1 built with classical and modified method

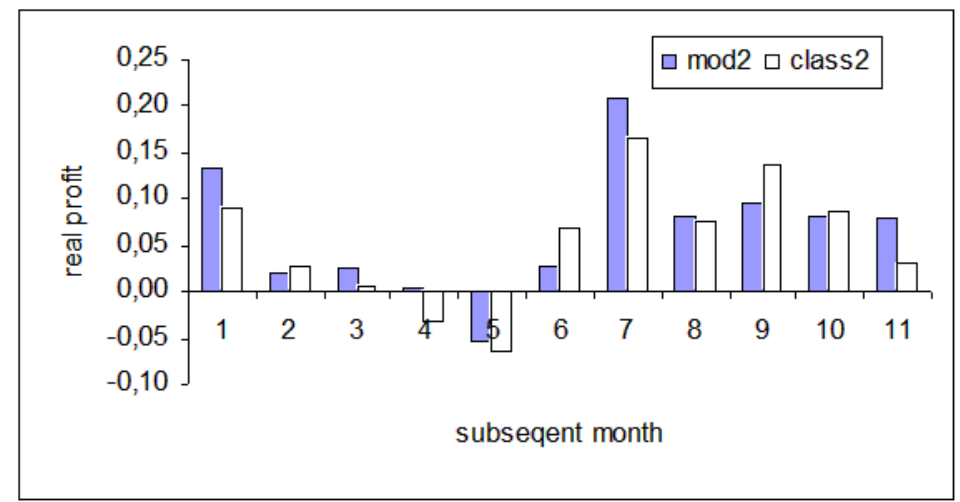

Fig. 3. Monthly profits for portfolio \#2 built with classical and modified method

\section{Third phase}

Ultimately we compared profits for portfolios build with both methods using consistent approach. To do this we compared two most aggressive classical portfolios with two "most robust" modified ones. The result of such a comparison is depicted in Figure 4. 


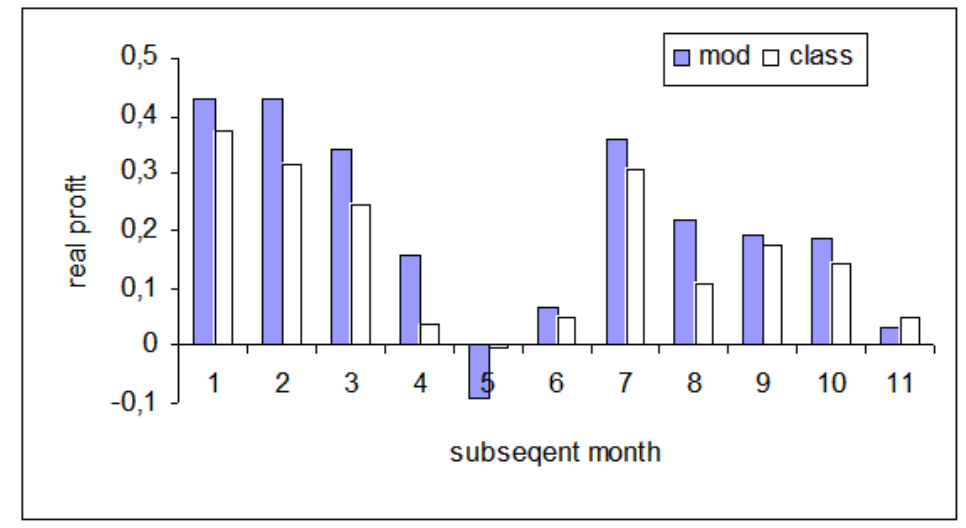

Fig. 4. Sums of monthly profits for two most aggressive portfolios built with classical and two most robust built with modified method

Additionally the analysis of portfolio based on 9 stocks, which are both aggressive and "robust" at the same time. Such an example portfolio consists of (debica, KGHM, krosno, orbis, PKNorlen, sokolow, TPSA, wolczanka, zywiec).

It appears that both methods construct quite different contents. Moreover for lambda values, for which there is no significant further portfolio diversification classical method "prefers" (in decreasing order): zywiec, krosno, debica, PKNorlen, sokolow; while the modified one: PKNorlen, zywiec, TPSA, sokolow.

For illustration the expected value and the variance of the portfolio being rebuilt during 14 months have been presented.

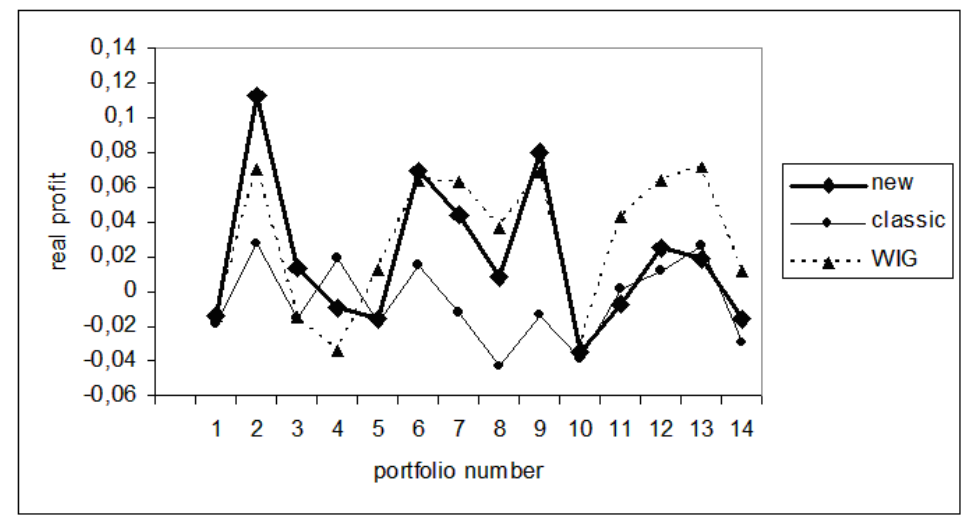

Fig. 5. Real profit comparison for both methods

To depict the difference in constructing models we present the real monthly return ratio for optimal contents for both portfolios for chosen $\lambda$ coefficient. The verification 
was done using past market price (exchange ratio) of the asset in the month, for which the prognosis was made. Figure 5 contains the comparison of real profits for both discussed methods. The WIG return $\mathrm{Rm}$ is also included as the background reference. It may be noticed that low-risk portfolio built using the proposed method gives per average profits greater than that of classic Sharpe's portfolio.

\section{CONCLUSIONS}

The modification of classical Sharpe's portfolio construction was proposed and its features were studied:

1. Classical and modified methods choose different asset shares for given portfolio.

2. The modified method gives higher yearly return for the year 2006 .

3. 3. Using strategy of strong portfolios construction based on assets with "robust" $\tilde{\beta}$ the resulting profit was averagely higher than that for classical strategy with aggressive assets.

4. The modified method is highly sensitive to local disturbances of monthly returns.

\section{REFERENCES}

1. O. Bunke, H. Bunke, Non Linear Regression, Functional Relationships, and Robust Methods, New York, Wiley, 1989.

2. N.R. Cox, The linear structural relation for several groups of data. Biometrika 63 (1976), $231-237$.

3. G.R. Dolby, The ultrastructural relation a synthesis of the functional and structural relations. Biometrika, 63 (1976), 39-50.

4. E.J. Elton, M.J. Gruber, Nowoczesna teoria portfelowa i analiza papierów wartościowych. WIG Press, Warszawa 1998.

5. W.A. Fuller, Measurement Error Models, Wiley, New York 1967.

6. H.M. Markowitz, Portfolio Selection, Journal of Finance, 7 (1952), 1, 77-91.

7. O. Reiersol, Identifiability of a linear relation between variables which are subject to error, Econometrica 18 (1950), 575-589.

8. W.F. Sharpe, Portfolio theory and capital markets, Mc Graw-Hill, New York 1970. 